\title{
Magnitude scales, category scales, and the general psychophysical differential equation
}

\author{
HENRY MONTGOMERY \\ University of Goteborg, Göteborg, Sweden
}

\begin{abstract}
The general psychophysical differential equation (GPDE) which relates scale values and Weber functions for two subjective variables was tested statistically with data from a number of category rating and magnitude estimation experiments. In all tests, it was investigated whether the GPDE is compatible with the assumption that the Weber function of the category scale is constant. This assumption implies that the category scale may be regarded as a discrimination scale. The tests were carried out by estimating the Weber function of the category scale by means of the GPDE and testing these estimates for constancy. For group data, there were fairly small systematic deviations from constancy across different experiments in the Weber functions that were estimated by the GPDE, and the hypothesis of a constant estimated Weber function could not be rejected statistically $(p>05)$ in most experiments. For individual data the estimated Weber functions deviated from constancy in idiosyncratic ways, and these deviations were statistically significant $(\mathrm{p}<.05)$ for most subjects.
\end{abstract}

In a series of studies, Eisler and his co-workers have demonstrated that the category scale is close to the Fechner integral of the Weber function of the magnitude scale (Eisler, 1962, 1963b c, 1965; Eisler \& Montgomery, 1974; Eisler \& Ottander, 1963; Montgomery \& Eisler, 1974). This is to say that the category scale is predicted by integrating the equation

$$
\frac{\mathrm{dK}}{\mathrm{d} \psi}=\frac{\mathrm{k}}{\sigma_{\psi}(\psi)}
$$

where $\mathrm{K}$ and $\psi$ denote, respectively, category and magnitude scale values for the same set of stimuli, $\mathrm{k}$ is a constant assumed to correspond to a constant Weber function for the category scale, and $\sigma_{\psi}(\psi)$ corresponds to the Weber function of the magnitude scale (usually computed as intraindividual SDs of the magnitude estimates). The form of the Weber function of the magnitude scale has been described as linear (Eisler, 1962, 1963b) or parabolic (Montgomery, 1975; Montgomery \& Eisler, 1974).

Equation 1 corresponds to a special case of the general psychophysical differential equation (the GPDE; Eisler, 1963a, 1965; Eisler, Holm, \& Montgomery, Note 1) which expresses the following relation

$$
\frac{\mathrm{dy}}{\mathrm{dx}}=\frac{\sigma_{\mathrm{y}}(\mathrm{y})}{\sigma_{\mathrm{x}}(\mathrm{x})},
$$

This work was supported by the Swedish Council for Social Science Research. I am indebted to Dr. Hanes Eisler for his very helpful comments on the manuscript. Requests for reprints should be sent to Henry Montgomery, Department of Psychology, University of Göteborg, Fack, S 40020 Göteborg 14, Sweden. where $\mathrm{x}$ and $\mathrm{y}$ denote scales values of subjective variables for the same set of stimuli, and $\sigma_{x}(x)$ and $\sigma_{\mathrm{y}}(\mathrm{y})$ their Weber functions. As can be seen, the Fechnerian model corresponds to the case with a constant $\sigma_{\mathrm{y}}(\mathrm{y})$ in Equation 2. This case of the GPDE is of particular interest since a constant $\sigma_{\mathrm{y}}(\mathrm{y})$ implies that the $y$ variable can be considered a discrimination scale provided certain other assumptions of the underlying scaling model are valid (cf. Eisler \& Montgomery, 1974; Torgerson, 1958, Chap. 10).

As mentioned above, the Fechnerian model allows good prediction of category scale values. From this it might be inferred that the GPDE is valid for the relationship between category and magnitude scales, and, furthermore, that the category scale is a dis. crimination scale. However, the assumption of a constant Weber function for the category scale seems to be contradicted by empirical results. The SDs for category ratings are typically greatest in the middle and decrease toward both ends. Eisler and Montgomery (1974) suggested that this discrepancy between theory and empirical data could be explained in terms of bias or distortion in the Weber function of the category scale. The primary cause of this bias was assumed to be that the more extreme stimuli are recognized more often than stimuli which are closer to the middle of the stimulus range (cf. Garner, 1952). In the Eisler and Montgomery (1974) study, an attempt was made to minimize this bias by (a) constructing a set of stimuli that were equally discriminable, and (b) constructing a set of stimuli with equal response ambiguity (Attneave, 1959) for all stimuli. For group data, the Weber function of the category scale proved approximately constant for both spacings except that the SDs corresponding to the two end categories were too low. For individual 
data, however, there were in some cases rather large deviations from constancy in the Weber functions of the category scale. The approximately constant Weber functions obtained for the category scales (particularly for group data) together with rather accurate predictions yielded by the GPDE and Fechnerian integration suggested a distinction between the genotypic, i.e., unbiased, Weber function (assumed to be constant for the category scale) and the phenotypic, i.e., empirically obtained, Weber function.

Thus, the Fechnerian model as well as the assumption of a constant genotypic Weber function of the category scale seem to be well supported by empirical results, particularly for group data. However, as will be shown below, objections can be raised against the procedures that have been used for testing the validity of the Fechnerian model (Equation 1) and the GPDE in general (Equation 2). The typical procedure in those previous tests has been to integrate Equations 1 or 2, to solve for one of the subjective variables, and to fit the equation obtained to empirical data. The computation of the integrals has been based on data that were collapsed over several subjects with an exception for a series of individual experiments in the Eisler and Montgomery (1974) study. The goodness of fit of these integrals has been evaluated only by eye or by purely descriptive measures. The following objections can be raised against this procedure:

(1) The procedure is insensitive. In the Eisler and Montgomery (1974) study, the fit of integrals of Equation 1 (the Weber function for the category scale assumed to be constant) and of Equation 2 (empirical Weber function for both subjective variables) were compared for a number of group and individual experiments on category rating and magnitude estimation. The difference in the fit of the two integrals proved small, despite the fact that the deviations from constancy in the Weber function for the category scale were occasionally rather large. Since integrating has a certain data smoothing consequence, thereby leading to a decrease in sensitivity, studying Equation 2 directly rather than after integration should give a clearer picture of the correctness of the GPDE (cf. Mashour, 1964).

(2) The use of data that were collapsed over several subjects is questionable, especially for the Weber function of the magnitude scale, since the form of this Weber function can vary markedly between individuals (Eisler \& Montgomery, 1974).

(3) It is difficult to draw more definitive conclusions about the goodness of fit of predictions allowed by the GPDE without using statistical tests.

The aim of the present study was to test the Fechnerian case of the GPDE in a way that takes the above objections into consideration. This was done for a number of group and individual experi- ments (cf. Table 1) by estimating the value of $k$ (Equation 1) for each pair of adjacent stimuli for each subject separately. In the individual experiments, the sampling variance of each $k$ value was estimated and $\mathbf{k}$ was tested for constancy by means of an $F$ test. In the group experiments, a nonparametric test (Friedman's two-way analysis of variance) was used for testing the hypothesis of constant $k$ values for all pairs of adjacent stimuli.

The data analysis outlined above require that the same subjects be employed for category rating and magnitude estimation and that individual data be available. The analysis was carried out with data from all experiments reported by Eisler and his coworkers that fulfilled these requirements. The experiments are described in some detail in Table 1 . As mentioned above, data from group and individual experiments were used. The group experiments represent four different continua, viz, loudness of white noise, softness of white noise, intensity of smell of amyl acetate, and length of lines. In the individual experiments, all subjects judged the loudness of white noise.

\section{DATA ANALYSIS}

\section{Estimation of $k$ Values}

Solving for $\mathrm{k}$ in Equation 1 yields

$$
\mathbf{k}=\frac{\mathrm{dK}}{\mathrm{d} \psi} \sigma_{\psi}(\psi) .
$$

In both the group and individual experiments, the value of $k$ in this equation was estimated for each subject separately, and for each pair of adjacent stimuli $n$ and $n+1$ according to

$$
\mathbf{k}^{\prime}=\frac{\mathbf{K}_{\mathrm{n}+1}-\mathbf{K}_{\mathrm{n}}}{\psi_{\mathrm{n}+1}-\psi_{\mathrm{n}}} \cdot\left(\frac{s_{\mathrm{n}+1}+s_{\mathrm{n}}}{2}\right)
$$

where $\mathrm{K}$ and $\psi$ denote arithmetic means of category ratings and magnitude estimates, and $s$ the sample standard deviation of the magnitude estimates.

The quantity $\left(K_{n+1}-K_{n}\right) /\left(\psi_{n+1}-\psi_{n}\right)$ in Equation 3 is an estimate of the derivative of the category scale with respect to the magnitude scale. It should be noted that there could be two kinds of errors in this estimate of the derivative, viz, systematic errors and stochastic errors.

An attempt to assess the systematic error was made with values from explicit functions obtained by integration of Equation 1 for two cases of combinations of Weber functions, viz, linear-constant and parabolic-constant. The numerical values of these functions were taken from two experiments on loudness of white noise, viz, Eisler (1962, linear-constant combination of Weber functions) and Montgomery 
Table 1

Experiments Employed in the Test of the Fechnerian Model (Equation 1)

Denota-

Group Experiments

tion in

\begin{tabular}{|c|c|c|c|c|}
\hline Text & Reference & Continuum & $\mathrm{N}_{\mathrm{c}}$ & Remarks \\
\hline Loud 1 & Eisler, 1962 & Loudness of white noise & 7 & 1: Log spacing, ambient noise not controlled \\
\hline Loud 2 & Eisler, 1962 & Loudness of white noise & 7 & \\
\hline Loud 3 & Eisler, 1962 & Loudness of white noise & 7 & \\
\hline Soft 1 & Eisler, 1962 & Sof tness of white noise & 7 & 2: Sone spacing, ambient noise not controlled \\
\hline Soft 2 & Eisler, 1962 & Softness of white noise & 7 & \\
\hline Soft 3 & Eisler. 1962 & Softness of white noise & 7 & 3: Log spacing, sound proofed chamber \\
\hline Smell 1 & Eisler, $1963 b$ & Intensity of smell of amyl acetate & 5 & 1-3: Different standards in magnitude estimation \\
\hline Smell 2 & Eisler, $1963 b$ & Intensity of smell of amylacetate & 5 & \\
\hline Smell 3 & Eisler, $1963 b$ & Intensity of smell of a myl ace tate & 5 & \\
\hline Line 1 & Eisler, $1963 c$ & Length of lines & 9 & Stimulus exposure $.12 \mathrm{sec}$ \\
\hline Line 2 & Eisler, $1963 c$ & Length of lines & 9 & Stimulus exposure as long as the subject wished \\
\hline ERA & Eisler \& Montgomery, 1974 & Loudness of white noise & 7 & Equal response ambiguity (ERA) spacing \\
\hline ED 1 & Eisler \& Montgomery, 1974 & Loudness of white noise & 7 & Equal discriminability (ED) spacing \\
\hline ED 2 & Eisler \& Montgomery, 1974 & Loudness of white noise & 15 & ED spacing \\
\hline
\end{tabular}

Initials

\begin{tabular}{|c|c|c|c|c|}
\hline of Ss & Reference & Continuum & $\mathrm{N}_{\mathrm{c}}$ & Remarks \\
\hline $\begin{array}{l}\text { K.J., D.M. } \\
\text { M.W.,B.J. }\end{array}$ & Eisler \& Montgomery, 1974 & Loudness of white noise & 7 & ERA spacing \\
\hline D.F., M.L. & Eisler \& Montgomery, 1974 & Loudness of white noise & 7 & ED spacing \\
\hline H.S., E.E. & Eisler \& Montgomery, 1974 & Loudness of white noise & 7 & $\begin{array}{l}\text { ED spacing, stimuli presented in ascending and } \\
\text { descending series }\end{array}$ \\
\hline $\begin{array}{l}\text { T.J., A.N. } \\
\text { B.E., I.S. }\end{array}$ & Eisler \& Montgomery, 1974 & Loudness of white noise & 15 & ED spacing \\
\hline
\end{tabular}
B.E., I.S.

Note $-N_{c}$ denotes the number of rating categories in cach category rating experiment.

and Eisler (1974, parabolic-constant combination of Weber functions). The difference between the estimated derivatives and derivatives computed by differentiating the expicit function was about $1 \%$ for practically all scale values in both combinations of Weber functions.

The stochastic error in the estimated derivatives was estimated by means of error calculus (cf. below under Statistical Analysis) for each subject and pair of adjacent stimuli in the individual experiments in the Eisler and Montgomery (1974) study. The median of the relative standard error

$$
\left(\frac{\frac{s_{d}}{K_{n+1}-K_{n}}}{\psi_{n+1}-\psi_{n}}\right),
$$

where $s_{d}$ denotes the standard error of the estimated derivatives, was 0.173 and the quartile deviation was 0.057. Thus, the stochastic error in the estimated derivatives was rather large. A more efficient estimate of the derivative could not be found, however. Spline derivatives (Greville, 1969) had still greater estimated stochastic errors for the present data, whereas derivatives computed by parabolic interpolation (Hildebrand, 1956) had estimated stochastic errors that varied on approximately the same level as the errors in those derivatives that were computed from Equation 3. However, the stochastic error for the latter estimates of the derivative seemed to be more homogeneous than for the derivatives that were computed by parabolic interpolation.

\section{Statistical Analysis}

Group experiments. For each group experiment, the hypothesis of constant $\mathrm{k}^{\prime}$ values for all pairs of adjacent stimuli was tested with Friedman's twoway analysis of variance (Subjects by Pairs of Adjacent Stimuli). No parametric test was used, since the distribution of $\mathbf{k}^{\prime}$ values were markedly skewed and heterogeneous.

Individual experiments. For each of the 12 subjects in the individual experiments (Eisler \& Montgomery, 1974), $k^{\prime}$ was tested for constancy by means of an $F$ test. The sampling variance in each $k^{\prime}$ value was estimated by using error calculus. The computations were based on the following equation (cf. Beers, 1953, p. 29).

$$
\begin{aligned}
s_{V}^{2}=\sum_{i=1}^{n}\left(\frac{\delta V}{\delta_{x_{i}}}\right)^{2} s_{x_{i}}^{2} & \\
& +2 \sum_{i=1}^{n} \sum_{j=1}^{n} r_{x_{i} x_{j}} \cdot \frac{d V}{\delta_{x_{i}}} \frac{\delta V}{d_{x_{j}}} s_{x_{i}} s_{x_{j}}
\end{aligned}
$$

where $s_{V}^{2}$ denotes the variance, $V$ is a function of 
the variables $x_{1}, x_{2} \ldots x_{n}$, and $r$ denotes the product moment correlation. For the present data, $s_{\mathrm{V}}^{2}$ corresponds to the sampling variance of $\mathrm{k}^{\prime}$ and $\delta \mathrm{V} / \delta_{\mathrm{xi}_{\mathrm{i}}}$ corresponds to the partial derivates of $\mathbf{k}^{\prime}$ with respect to the variables on the right-hand side of Equation 3. These partial derivatives can be obtained by logarithmic differentiation of Equation 3 (cf. Hald, 1952, p. 250). Differentiating

$$
\begin{aligned}
\ln k^{\prime}= & \ln \left(K_{n+1}-K_{n}\right)-\ln \left(\psi_{n+1}-\psi_{n}\right) \\
& +\ln \left(s_{n+1}+s_{n}\right)-\ln 2
\end{aligned}
$$

yields

$$
\begin{gathered}
\frac{1}{\mathbf{k}^{\prime}} \cdot \frac{d \mathbf{k}^{\prime}}{\delta \mathbf{K}_{\mathrm{n}+1}}=\frac{1}{\mathbf{K}_{\mathrm{n}+1}-\mathbf{K}_{\mathrm{n}}}, \\
\frac{1}{\mathbf{k}^{\prime}} \cdot \frac{\delta \mathbf{k}^{\prime}}{\delta \mathbf{K}_{\mathrm{n}}}=\frac{1}{\mathbf{K}_{\mathrm{n}+1}-\mathbf{K}_{\mathrm{n}}}, \\
\frac{1}{\mathbf{k}^{\prime} \cdot \frac{\delta \mathbf{k}^{\prime}}{\delta \psi_{\mathrm{n}+1}}}=-\frac{1}{\psi_{\mathrm{n}+1}-\psi_{\mathrm{n}}}, \\
\frac{1}{\mathbf{k}^{\prime}} \cdot \frac{\delta \mathbf{k}^{\prime}}{\delta \psi_{\mathrm{n}}}=\frac{1}{\psi_{\mathrm{n}+1}-\psi_{\mathrm{n}}}, \\
\frac{1}{\mathbf{k}^{\prime}} \cdot \frac{\delta \mathbf{k}^{\prime}}{\delta \mathrm{s}_{\mathrm{n}+1}}=\frac{1}{\mathrm{~s}_{\mathrm{n}+1}+\mathrm{s}_{\mathrm{n}}},
\end{gathered}
$$

and

$$
\frac{1}{k^{\prime}} \cdot \frac{\delta k^{\prime}}{\delta s_{n}}=\frac{1}{s_{n+1}+s_{n}}
$$

The following assumptions were made about the correlations between the variables in Equation 3. The correlation between the $K$ and $\psi$ variables and between $K$ and $s$ were assumed to be zero since $K$ and $\psi$ derive from different experiments. The correlation between the arithmetic means and the standard deviations of the same samples of observations will be zero if the observations are normally distributed (cf. Cramér, 1945, p. 382). Since the magnitude estimates were approximately normally distributed in the individual experiments, it was assumed that $r_{\psi_{n+1} s_{n+1}}$ and $r_{\psi_{n} s_{n}}$ were approximately zero. Because of this assumption, it seemed reasonable also to assume that $r_{\psi_{n+1} s_{n}}$ and $r_{\psi_{n} s_{n+1}}$ were approximately zero. Finally, the covariance term corresponding to $r_{S_{n+1}}$ was neglected since the numerical values of

$$
\frac{\delta \mathbf{k}^{\prime}}{\delta s_{n+1}} \cdot \frac{d \mathbf{k}^{\prime}}{\delta s_{n}} s_{n+1} \cdot s_{n}
$$

proved to be very small compared to the other error terms.

The above assumptions imply that only two of the covariance terms need to be computed, i.e., the terms corresponding to $r_{K_{n+1} K_{n}}$ and $r_{\psi_{n+1} \psi_{n}}$. Inserting these terms as well as the variance terms corresponding to $K_{n}, K_{n+1}, \psi_{n}, \psi_{n+1}, s_{n}$, and $s_{n+1}$ into Equation 4 yields, after rearranging,

$$
\begin{aligned}
s_{k^{\prime}}^{2}= & \left(\frac{k^{\prime}}{K_{n+1}-K_{n}}\right)^{2}\left(s_{K_{n+1}^{2}}^{2}+s_{K_{n}}^{2}\right. \\
& \left.-2 r_{K_{n+1} K_{n}} \cdot s_{K_{n+1}} \cdot s_{K_{n}}\right) \\
& +\left(\frac{k^{\prime}}{\psi_{n+1}-\psi_{n}}\right)^{2}\left(s_{\psi_{n+1}}^{2}+s_{\psi_{n}}^{2}\right. \\
& \left.-2 r_{\psi_{n+1} \psi_{n}} \cdot s_{\psi_{n+1}} \cdot s_{\psi_{n}}\right) \\
& +\left(\frac{k^{\prime}}{s_{n+1}+s_{n}}\right)^{2}\left(s_{S_{n+1}^{2}}^{2}+s_{S_{n}}^{2}\right) .
\end{aligned}
$$

As can be seen from Equation 12, the above assumptions imply that the error variance of $\mathbf{k}^{\prime}$ can be partitioned into three additive components which are related to the variances of $K_{n+1}-K_{n}$, $\psi_{n+1}-\psi_{n}$, and $\left(s_{n+1}+s_{n}\right) / 2$, respectively.

The error variance of $k^{\prime}$ was estimated according to Equations 12 for each individual and for each pair of adjacent stimuli. In the following discussion, this estimate of $s_{k^{\prime}}^{2}$ is denoted as $s^{\prime 2} k^{\prime}$. As noted above, $K_{n}, K_{n+1}, \psi_{n}$, and $\psi_{n+1}$ correspond to arithmetic means of single observations. The variance of these arithmetic means was estimated according to $s^{2} /(N-1)$, where $N=$ number of trials, whereas the variance of $s_{n}$ and $s_{n+1}$, respectively, was estimated according to $\mathrm{s}^{2} /[2(\mathrm{~N}-1)]$ (cf. McNemar, 1950, p. 60). The estimation of $r_{K_{n+1} K_{n}}$ and $r_{\psi_{n}+1 \psi_{n}}$ was dependent on the order in which the stimuli were presented. For 10 of the 12 subjects the 10 different stimuli were presented in 50 successive blocks, where each block consisted of all 10 stimuli presented in random order. The correlations for these 10 subjects were computed between the single observations from the two stimuli within each pair of adjacent stimuli over all blocks of stimuli. [Note that correlations between single observations can be 
expected to be equivalent to correlations between arithmetic means (Beers, 1953, p. 31)]. For the two remaining subjects (subjects H.S. and E.E.), the 10 different stimuli were presented in 84 successive blocks of ascending or descending stimuli, where each block consisted of 5, 6, or 7 stimuli (cf. Eisler \& Montgomery, 1974; Eisler \& Ottander, 1963). For these two subjects, $\mathbf{r}_{\mathbf{K}_{n+1} K_{n}}$ and $\mathbf{r}_{\psi_{n+1} \psi_{n}}$ were computed between arithmetic means of samples of observations from stimulus $n$ and $n+1$, respectively, where each sample consisted of seven blocks which were drawn randomly without replacement until all blocks had been drawn. The correlations were thus computed over 12 samples of observations between the means of the observations from the two stimuli within each pair of adjacent stimuli.

As mentioned above, the hypothesis of constant $k^{\prime}$ values was tested by means of an $F$ test for each of the 12 subjects in the individual experiments. This $F$ test was based on the ratio between the estimated population variance of $k^{\prime}$ between the nine different pairs of adjacent stimuli and the arithmetic mean of $\mathrm{s}^{\prime}{ }^{2}$, (Equation 12) within the nine different pairs of adjacent stimuli. The degrees of freedom for the within and between variances, respectively, were assumed to be 441 and 8 . (The total number of observations was considered to be 450 , since there were 9 pairs of adjacent stimuli and 50 observations from each stimulus in the category rating and magnitude estimation task, respectively.)

It can be noted that the above procedure for testing the Fechnerian case of the GPDE (Equation 1) involves two steps: (a) estimating values of $\mathrm{k}$ in Equation 1 and (b) conducting an $F$ test of the hypothesis that these estimated $k$ values are constant. In order to check the adequacy of this procedure for testing Equation 1, a computer simulation was carried out. The simulation was based on data from three subjects (B.J., M.L., and T.J.) which were used for constructing cases in which Equation 1 yielded constant population values of $\mathbf{k}$ for all nine pairs of adjacent stimuli. The population values of $\psi$ (mean magnitude estimates) and of $s_{K}$ (SDs of category ratings) were the same as the corresponding empirically obtained values for the three subjects. The population values of $s_{\psi}$, i.e., $\sigma_{\psi}$ in Equation 1, were obtained by letting $\sigma_{\psi}(\psi)$ be defined by a parabola which was fitted to the empirical Weber function of the magnitude scale for each of the three subjects. The population values of $\mathrm{K}$ (mean category ratings) were derived by inserting the fitted parabolas into Equation 1 and solving the equation for $K$. The "category ratings" and "magnitude estimations" in these constructed cases were normally distributed, and the population values of the correlations $r_{\psi_{11+1} \psi_{n}}$ and $\mathbf{r}_{K_{n+1}} K_{n}$ were the same as the corresponding empirical correlations for the three subjects. From these distributions, a distribution of $1,000 \mathrm{~F}$ ratios was generated for each case by drawing random samples of 50 "category ratings" and "magnitude estimations," respectively, and computing the $F$ ratio for each sample over all the nine pairs of adjacent stimuli. The distributions of $F$ ratios thereby generated was then compared to the $F$ distribution that obtains for the degrees of freedom that were used in the test described above. It was found that the 95th and 99th percentiles in these F distributions corresponded to the $93.4,96.7$, and 94.3 percentiles and the $97.6,99.0$, and 98.2 percentiles, respectively, in the simulated distributions. Consequently, the results of the simulation suggest that the present test of the Fechnerian case of the GPDE is valid within acceptable limits.

\section{RESULTS}

\section{Group Experiments}

Figure 1 shows for each group experiment medians of the $\mathrm{k}^{\prime}$ values as a function of the category scale (cf. the circles in Figure 1). The medians were taken over all subjects for each pair of adjacent stimuli and are in the following discussion denoted as $\mathbf{M d}_{\mathrm{k}}$. The horizontal straight lines in Figure 1 represent medians of $\mathbf{M d}_{\mathrm{k}^{\prime}}$ over all pairs of adjacent stimuli. In most experiments, there is a considerable variability in $\mathbf{M d}_{\mathbf{k}^{\prime}}$ around this horizontal line. For almost all experiments, the $\mathbf{M d}_{\mathrm{k}^{\prime}}$ values for the extreme pairs of stimuli lie below the horizontal line. No other common trend can be seen in the deviations of $\mathrm{Md}_{\mathrm{k}^{\prime}}$ from the horizontal line.

Friedman's two-way analysis of variance indicated a significant effect of pairs of adjacent stimuli on $\mathrm{k}^{\prime}(\mathrm{p}<.05)$ in 5 of the 14 experiments (cf. Table 2$)$, viz, in Experiments Loud 2, Soft 2, Line 1, ED 1, and ED 2. The strongest significant effect was obtained in Experiment ED 2, in which the subjects rated the stimuli on a 15 -point category scale. The $\mathbf{M d}_{\mathbf{k}}$, values in this experiment tend to increase from the lower end of the category scale toward a point corresponding to the 10th rating category and then to decrease toward the upper end of the category scale. This result corroborates the tentative conclusion drawn in the Eisler and Montgomery (1974) study that the 15-point category scale is not a discrimination scale in the sense of having a constant genotypic Weber function.

The significant effects in Experiments Loud 2, Soft 2, and ED 1 are probably at least partially due to the fact that some stimuli were closely bunched in these experiments, particularly the stimuli in the louder region of the stimulus range. This is because these stimuli were so close together that many subjects gave the same category ratings for adjacent stimuli, which implies that $\mathrm{k}^{\prime}$ became zero in these 

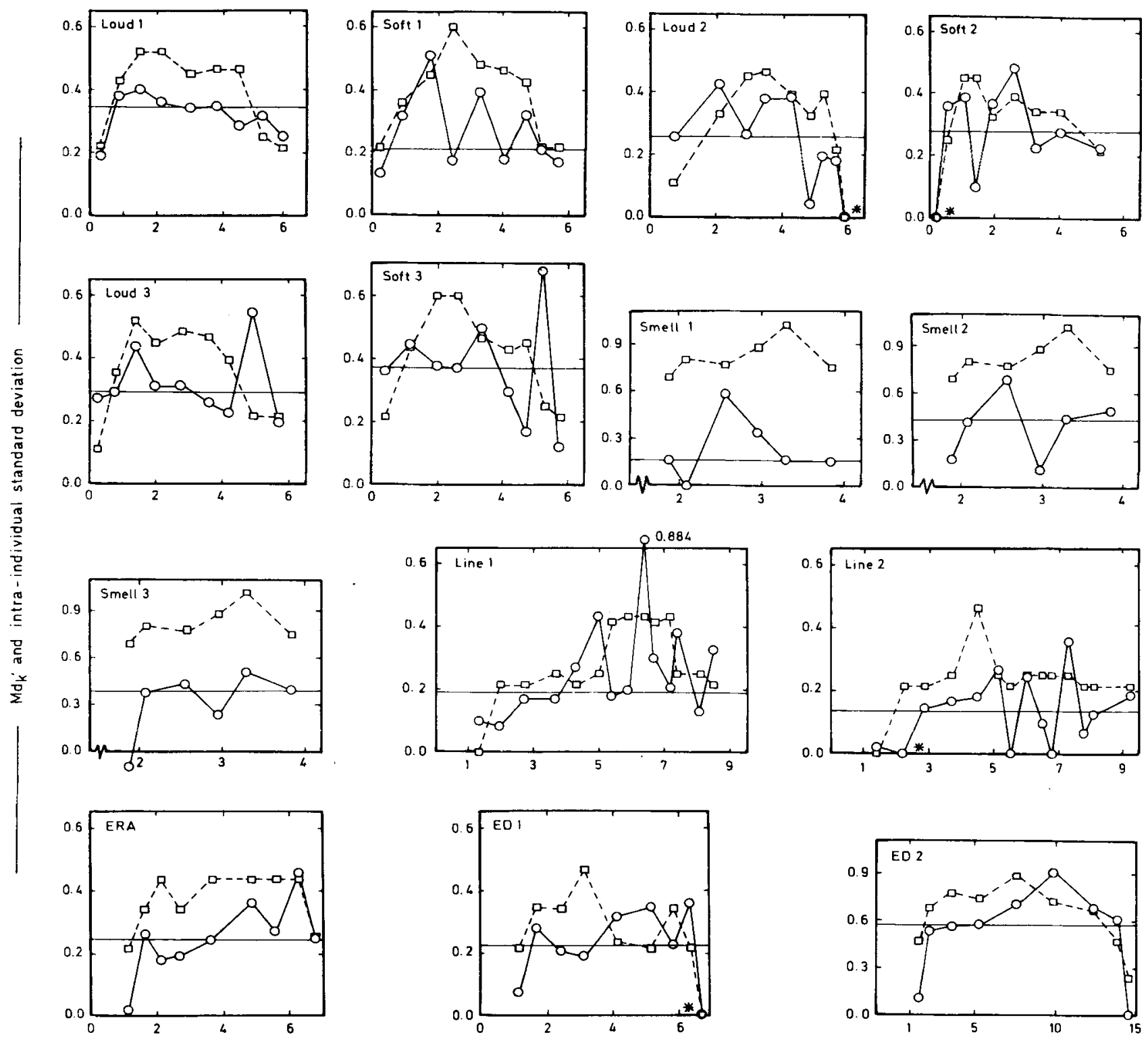

Figure 1. Theoretical and empirical measures of the uncertainty in category ratings as a function of the corresponding category scale (arithmetic means of the two category scale values from each pair of adjacent stimuli) for the group experiments listed in Table 1 [circles: medians of $k^{\prime}$ (Equation 3) over all subjects for each pair of adjacent stimuli $\left(\mathbf{M D}_{\mathbf{k}^{\prime}}\right.$ ); squares: intraindividual SDs]. The asterisks correspond to those pairs of adjacent stimuli for which $k^{\prime}$ was equal to zero for more than $50 \%$ of the subjects. The straight horizontal line in each panel represents the median of $\mathbf{M d}_{\mathbf{k}}$, over all pairs of adjacent stimuli.

cases, provided that $\psi_{\mathrm{n}+1}-\psi_{\mathrm{n}}$ was different from zero, which was practically always the case. (Note that the subjects were only allowed to use integers in the category rating task.) The points in Figure 1 that are marked with an asterisk correspond to those cases in which $\mathrm{k}^{\prime}$ was equal to zero for more than $50 \%$ of the subjects. If the points marked with an asterisk are excluded, there is a barely significant effect of pairs of adjacent stimuli on $\mathbf{k}^{\prime}$ in Experiment Soft 2 and no significant effect in the other two experiments (cf. Table 2).

The significant effect in Experiment Line 1 is more difficult to account for. There were relatively few cases of $k^{\prime}=0$ in this experiment, and the function which relates $\mathrm{Md}_{\mathrm{k}}$, to the category scale is most irregular. This function bears some resemblance to the corresponding function in Experiment Line 2, in which an almost significant effect was obtained. 
Table 2

Friedman's Two-Way Analysis of Variance of $k^{\prime}$ (Equation 3) as a Function of Pair of Adjacent Stimuli in the Group Experiments Listed in Table 1

\begin{tabular}{lcc}
\hline Experiment & df & \multicolumn{1}{c}{$\chi^{2}$} \\
\hline Loud 1 & 8 & 10.31 \\
Soft 1 & 8 & 9.54 \\
Loud 2 & 8 & $22.90^{* *}$ \\
Loud 2 & 7 & 13.88 \\
Soft 2 & 8 & $23.12^{* *}$ \\
Soft 2 $\dagger$ & 7 & $14.50^{*}$ \\
Loud 3 & 8 & 7.80 \\
Soft 3 & 8 & 15.50 \\
Smell 1 & 5 & 4.16 \\
Smell 2 & 5 & 5.75 \\
Smell 3 & 5 & 3.90 \\
Line 1 & 13 & $25.72^{*}$ \\
Line 2 & 13 & 22.51 \\
ERA & 8 & 8.82 \\
ED 1 & 8 & $18.32^{*}$ \\
ED 1 + & 7 & 14.06 \\
ED 2 & 8 & $26.81^{* *}$ \\
\hline
\end{tabular}

TPairs of stimuli for which $k$ ' was equal to zero for more than $50 \%$ of the subjects excluded from the test. ${ }^{*} p<.05{ }^{* *} p<.01$

It can be noted that the Weber function of the magnitude scales in the two experiments on length of line was linear only for the lower part of the stimulus range, and that the $\psi$ intercept was close to zero. Similar Weber functions were also obtained in Experiments ED 1 and ED 2, but the relation between the category and magnitude scale was much more curvilinear in these two experiments than in the experiments on length of line.

Estimates of $\mathbf{k}$ were also computed from group data values of the variables in Equation 3 (geometric means of the arithmetic means of the magnitude estimates from each subject, arithmetic means of the category ratings over all subjects, and intraindividual SDs computed as described in Eisler, 1962). Apart from being somewhat greater than $\mathbf{M d}_{\mathrm{k}}$, there were no clearly systematic deviations between these estimates of $\mathrm{k}$ (not shown here) and the medians of the individual $k^{\prime}$ values.

The squares in Figure 1 correspond to intraindividual SDs of the category ratings as a function of the category scale. These SDs were computed in a way that was analogous to the computation of ' $\mathrm{k}^{\prime}$ and $\mathbf{M d}_{\mathrm{k}}{ }^{\prime}$. For each pair of adjacent stimuli $\mathrm{n}$ and $n+1$, the quantity $\left(s_{K}+s_{K_{n+1}}\right) / 2$, where $K$ denotes category ratings and $s$ standard deviation, was computed for each subject, and the median of this quantity was taken over all subjects for each pair of adjacent stimuli. In all experiments, the intraindividual SDs tend to be greater than $\mathrm{Md}_{\mathrm{k}}$. This tendency is most conspicuous in the smell experiments.

In Figure 2, an attempt was made to summarize the results from all experiments on loudness or softness, except Experiment ED 2 (the only experiment with a 15-point category scale). The ordinate values in Figure 2 were obtained by taking medians across different experiments of $\mathrm{Md}_{\mathrm{k}}$ and intraindividual SDs of category ratings, respectively, and smoothingthese medians over three successive values (moving averages). In panel $\mathrm{A}$ of Figure 2, the medians were obtained by rank ordering the category scale values in each experiment and then taking the median over all pairs of adjacent stimuli with the same ranks. (The number of stimuli was 10 in all experiments.) The medians corresponding to panel $\mathrm{B}$ were obtained by rank-ordering the arithmetic means of the two category scale values from each pair of adjacent stimuli across all eight experiments and then taking the median over the eight values corresponding to ranks 1-8, 9-16, etc., to ranks 65-72. (The category scale values in Experiments ERA and ED 1 were first reduced by 1 in order to make the category scales in these experiments cover the same range of numbers as the category scales in the other experiments.)

Both panels of Figure 2 show the same trends: The averages of $\mathbf{M d}_{\mathrm{k}}$ ' are very close to constancy but with somewhat too low values at the extremes of the category scale. The averages of the intraindividual SDs are greater than the averages of $\mathrm{Md}_{\mathbf{k}}$ ' and decrease from the middle of the stimulus range toward both ends.

\section{Individual Experiments}

Figure 3 shows, for each subject in the individual experiments, $\mathrm{k}^{\prime}$ as a function of the category scale (cf. the circles in Figure 3). The horizontal straight line in each panel represents the median of the $k^{\prime}$ values over all pairs of adjacent stimuli. As in the group experiments, there is a considerable variability in the estimates of $k$ around the horizontal line. However, in contrast to the group experiments, there is no common trend in the deviations from the horizontal line. It can also be seen from Figure 3 that the $k^{\prime}$ values vary within approximately the same region as the SDs of the category ratings (cf. the squares in Figure 3).

The estimates of the sampling error in $\mathrm{k}^{\prime}\left(\mathrm{s}_{\mathrm{k}}^{\prime}\right)$ as well as the empirical SDs of the category scale $\left(s_{K}\right)$ are introduced in Figure $\mathbf{3}$ as vertical lines around

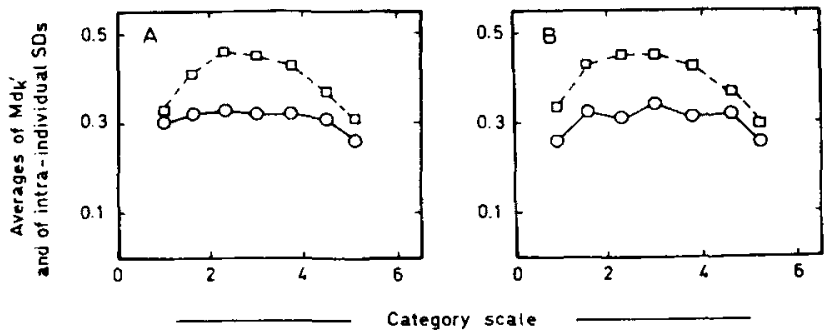

Figure 2. Theoretical and empirical measures of the uncertainty in category ratings as a function of arithmetic means of category scale values over all experiments on loudness or softness except Experiment $\mathbf{E D} 2$ (circles: averages of $\mathbf{M d}_{\mathrm{k}}$; squares: averages of intraindividual SDs). 

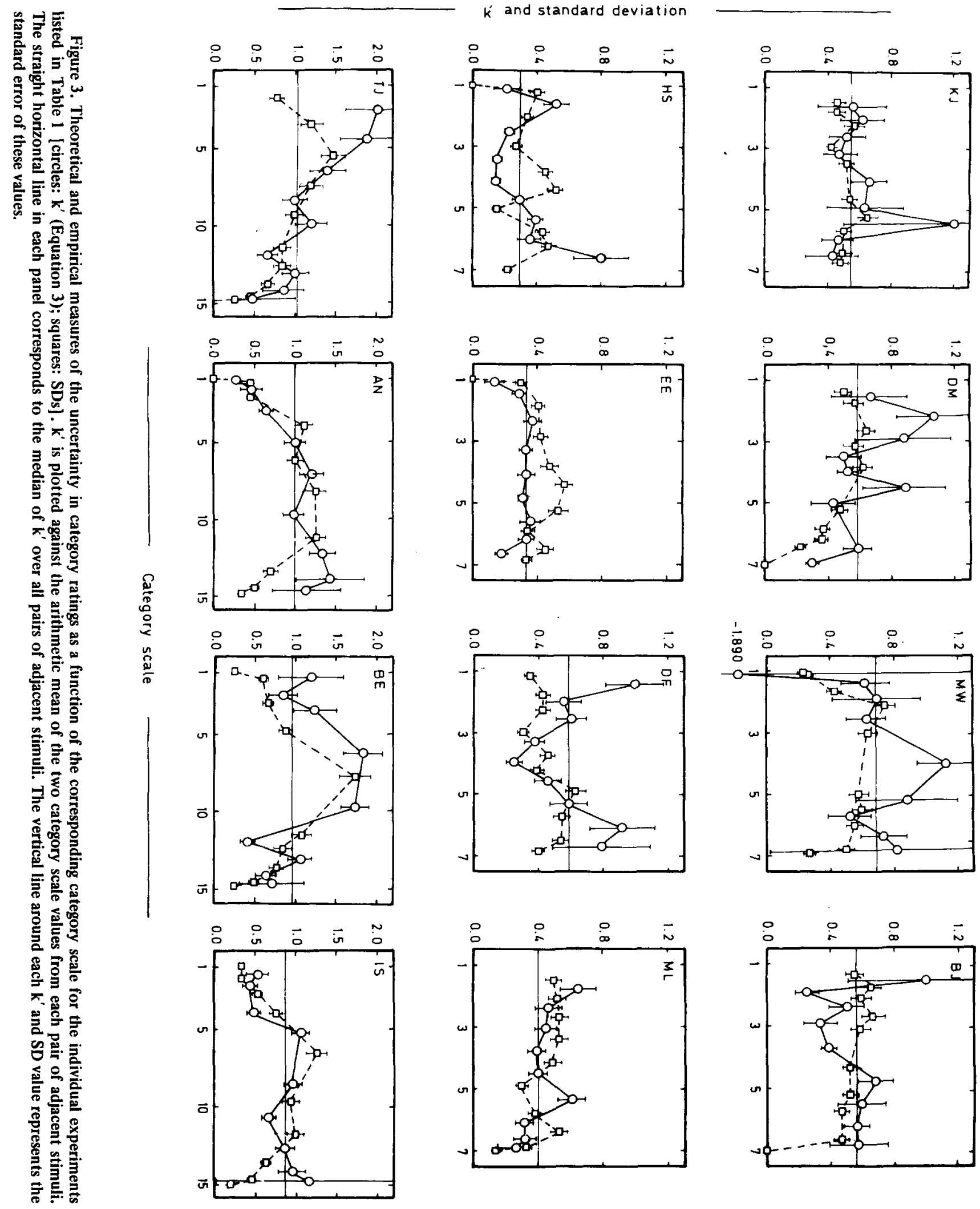
each value of $\mathrm{k}^{\prime}$ and $\mathrm{SD}$, respectively [cf. also the within variances $\left(s_{w}^{2}\right)$ in Table 3]. It can be seen that $s_{\mathbf{k}^{\prime}}^{\prime}$ varies considerably from subject to subject. In some cases, there is also a considerable intraindividual variation in $s_{\mathrm{k}}^{\prime}$ ' (e.g., subjects D.M., M.W., and D.F.). ${ }^{1}$

Table 3 shows that there was a significant effect $(p<.05)$ of pairs of adjacent stimuli on $k^{\prime}$ for 8 of the 12 subjects. It should be noted, however, that 3 of these 8 subjects used a 15 -point category scale, which is probably not a pure discrimination scale (cf. Eisler \& Montgomery, 1974). No general trends can be seen in the deviations from constancy in $\mathrm{k}^{\prime}$. For individual subjects, the following trends can be noted: decreasing toward the upper end of the category scale (subject T.J.), increasing toward the upper end of the category scale (subject A.N.), U-shape (subject D.F. and possibly subject H.S.), and inverted U-shape (subjects M.W. and B.E.).

Figure 3 demonstrates that these individual trends in the $\mathbf{k}^{\prime}$ values for some subjects seem to be paralleled by corresponding trends in the empirical SDs of the category scale (cf. subjects D.M., M.W., E.E., T.J., and B.E.). Table 3 gives the product moment correlations between $k^{\prime}$ and $\left(s_{K_{n+1}}+s_{K_{n}}\right) / 2$. As can be seen, these correlations are positive for 10 out of the 12 subjects and significant $(p<.05)$ for 4 subjects. It merits attention, however, that for some subjects there seem to be clearly systematic deviations between $\mathrm{k}^{\prime}$ and the empirical SDs of the category scale (cf. particularly subjects H.S. and D.F.).

\section{DISCUSSION}

In most group experiments, the Fechnerian case of the GPDE could not be rejected statistically. However, there may have been fairly great sampling errors in the theoretical uncertainties $\left(\mathrm{Md}_{\mathrm{k}^{\prime}}\right)$ that were estimated by means of the GPDE, since, when the $\mathbf{M d}_{\mathrm{k}}$ ' values did not deviate significantly from constancy, there was generally a considerable variability in these values across different pairs of adjacent stimuli. On the other hand, there were fairly small systematic deviations from constancy in the $\mathbf{M d}_{\mathrm{k}}$, values across different experiments (cf. Figure 2). From this, it might be tentatively concluded that the Fechnerian case of the GPDE is at least approximately valid for group data from category ratings and magnitude estimation experiments, and, moreover, that a category scale may be close to a discrimination scale when the scale values are collapsed over several subjects.

For individual subjects, there were significant idiosyncratic deviations from constancy in the estimates of $k$. That is, the Fechnerian model was disconfirmed for most subjects in the individual experiments. Thus, the present test of the GPDE
Table 3

Statistical Analysis of $k^{\prime}$ (Equation 3) as a Function of Pair of Adjacent Stimuli in the Individual Experiments Listed in Table 1

\begin{tabular}{lrll}
\hline Subject & \multicolumn{1}{c}{$\mathrm{s}_{\mathrm{w}}^{2}$} & \multicolumn{1}{c}{$\mathrm{J}$} & \multicolumn{1}{c}{$\mathrm{I}$} \\
\hline K.J. & .075 & .65 & $.710^{*}$ \\
D.M. & .040 & $2.12^{*}$ & $.718^{*}$ \\
M.W. & 10.104 & .01 & .506 \\
B.J. & .039 & 1.10 & -.156 \\
H.S. & .006 & $5.96^{* * *}$ & -.007 \\
E.E. & .002 & $2.28^{*}$ & $.766^{*}$ \\
M.L. & .008 & $1.97^{*}$ & .306 \\
D.F. & .025 & $2.25^{*}$ & .177 \\
T.J. & .063 & $4.25^{* * *}$ & $.687 *$ \\
A.N. & .050 & $2.79^{* *}$ & .513 \\
B.E. & .049 & $4.37^{* * *}$ & .577 \\
I.S. & .071 & .89 & .274 \\
\hline
\end{tabular}

Note-df for all F tests $=8,441 ;$ df for all $r$ values $=8$.

${ }^{*} p<.05 \quad{ }^{* *} p<.01 \quad * * 0<.001$

does not generally support the assumption that category scales for individual subjects are pure discrimination scales in the sense of having a constant genotypic, i.e., unbiased, Weber function which can be predicted by means of the GPDE.

It might be speculated that the individual trends in the $\mathrm{k}^{\prime}$ values reflects corresponding trends in the genotypic Weber functions of the category scale. This would be the case if the GPDE is valid for genotypic Weber functions and if the empirical Weber function of the magnitude scale coincides with the genotypic Weber function, i.e., is free from bias. In this connection, it is interesting to note that the $\mathbf{k}^{\prime}$ values in the individual experiments tended to be positively correlated with the empirical SDs of the category ratings. Thus, the GPDE in its general form (Equation 2) accounted for part of the variance in the empirical SDs of the category ratings in the individual experiments. If the $\mathrm{k}^{\prime}$ values reflect the form of the genotypic Weber function, then this result suggests that the genotypic Weber function of category scales for individual subjects may covary with the corresponding phonotypic, i.e., empirical Weber function. It should be noted, however, that for group data as well as for some individual data there seemed to be clearly systematic deviations between phenotypic Weber functions and Weber functions that were predicted by the GPDE (cf. Figure 2 and Figure 3, subjects H.S. and D.F.).

It can be concluded that neither the Fechnerian model (Equation 1) nor the GPDE in general (Equation 2) may be strictly valid for individual category rating and magnitude estimation data. It should be borne in mind, however, that predictions of category scale values for individual subjects from the Fechnerian model or from the GPDE in general have been found to be close to empirical category scale values (Eisler \& Montgomery, 1974). Thus, although the GPDE may not be strictly valid for individual category and magnitude scales, it may still 
be a powerful instrument for predicting the relation between the two types of scales for individual subjects.

\section{REFERENCE NOTE}

1. Eisler, H., Holm, S., \& Montgomery, H. Is the general psychophysical differential equation an approximation? Reports from the Psychological Laboratories, University of Stockholm, 1973, No. 386.

\section{REFERENCES}

AttNeave, F. Applications of information theory to psychology. New York: Holt. Rinehart, \& Winston, 1959.

BeERs, Y. Theory of error. Reading, Palo Alto, \& London: Addison-Wesley, 1953.

CRAMÉr, H. Mathematical methods of statistics. Uppsala: Almqvist \& Wiksell, 1945.

EISLER, H. Empirical test of a model relating magnitude and category scales. Scandinavian Journal of Psychology, 1962, 4. $88-96$.

EIsLER. H. A general differential equation in psychophysics: Derivation and empirical test. Scandinavian Journal of Psychology, $1963,4,265-272$. (a)

EISLER. H. How prothetic is the continuum of smell? Scandinavian Journal of Psychology, 1963, 4, 29-32. (b)

Eisler, H. Magnitude scales, category scales, and Fechnerian integration. Psychological Review, 1963, 70, 243-252. (c)

EISLER, H. On psychophysics in general and the general psychophysical differential equation in particular. Scandinavian Journal of Psychology, 1965, 6, 85-102.

Eisler. H., \& Montgomery, H. On theoretical and realizable ideal conditions in psychophysics: Magnitude and category scales and their relation. Perception \& Psychophysics, 1974, 16. 157-168.
Eisler, H., \& QTtander, C. On the problem of hysteresis in psychophysics. Journal of Experimental Psychology, 1963, 65 , 530-536.

GARNER, W. R. An equal discriminability scale for loudness judgments. Journal of Experimental Psychology, 1952, 43, 232-238.

GREVILLE, T. N. E. Spline functions, interpolation and numerical quadrature. In A. Ralstone \& H. S. Wilf (Eds.), Mathematical methods for digital computers. New York: Wiley, 1969.

HALD, A. Statistical theory with engineering applications. New York: Wiley, 1952.

HILDEBRAND, F. B.Introduction to numerical analysis. New York: McGraw-Hill. 1956.

MASHOUR, M. On Eisler's general psychophysical differential equation and his Fechnerian integration. Scandinavian Journal of Psychology, 1964, 5, 225-233.

MCNemar, Q. Psychological statistics. New York: Wiley, 1950.

Montgomery, H. Direct estimation: Effect of methodological factors on scale type. Scandinavian Journal of Psychology, 1975, 16, 19-29.

Montgomery, H., \& Eisler, H. Is an equal interval scale an equal discriminability scale? Perception \& Psychophysics, 1974, $15,441-448$.

TORGERSON, W. S. Theory and methods of scaling. New York: Wiley, 1958.

\section{NOTES}

1. The stimulation of test of the GPDE indicated that this test is relatively insensitive to heterogeneous variances. The range of variation for the population values of $s_{k}^{2}$, (Equation 12) in the three simulated cases was $0.007-0.142,0.007-0.074$, and $0.017-0.312$, respectively.

(Received for publication July 13, 1976; revision accepted November $14,1976$. 